\title{
0 cuidado à pessoa em sofrimento mental: sob a ótica dos familiares
}

RESUMO | Objetivo: analisar a percepção do cuidado a pessoa com sofrimento mental sob a ótica dos familiares. Método: A pesquisa é exploratória-descritiva, com abordagem qualitativa. Para coleta, foi realizada a técnica de coleta de dados usando o grupo focal, utilizando questões norteadoras, no período de abril, ano de 2014. A amostra foi composta por 20 familiares/ cuidadores de usuários do CAPS I, na capital Paraibana. Os dados qualitativos foram analisados, tendo-se como referência o método de análise de conteúdo, do tipo categorial temática. Resultados: Foram identificadas as seguintes categorias: Categoria I - Interferência e convivência com a doença mental e a Categoria II - A sobrecarga no cuidado ao doente mental. Conclusão: Considera-se, que ainda existam lacunas a serem preenchidas para se efetivar uma oferta qualificada a estes atores (usuários/ cuidadores-familiares) que, de fato, possam empoderar estes sujeitos e minimizar seus sofrimentos.

Palavras-chaves: Sofrimento; Saúde Mental; Cuidado; Família.

\begin{abstract}
Objective: to analyze a perception of caring for a person with mental distress and two family members sobbing. Method: The research is exploratory-descriptive, with a qualitative approach. For collection, the data collection technique was performed using the focus group, using guiding questions, in the period of April, 2014. It was shown to be composted by 20 family members / caregivers of CAPS I users. The qualitative data are analyzed, they are tended as a reference or method of content analysis, of thematic category type. Results: identified as following categories: Na Category I - Interference and coexistence with a mental education and a Category II - A overload not caring for the mental patient. Conclusion: It is considered that there are still lacunae to be pre-filled to make a qualified offer to these actors (users / caregivers-relatives) that, of course, will be able to empower these little subjects and minimize their.
\end{abstract}

Keywords: Hurt; Mental Health; Careful; Family.

RESUMEN | Objetivo: analizar la percepción del cuidado de las personas con sufrimiento mental desde la perspectiva de los familiares. Método: La investigación es exploratoria-descriptiva, con enfoque cualitativo. Para la recolección, la técnica de recolección de datos se realizó mediante el grupo focal, mediante preguntas orientadoras, en el período de abril de 2014 . La muestra estuvo formada por 20 familiares / cuidadores de usuarios de CAPS I. Se analizaron datos cualitativos, teniendo como referencia el método de análisis de contenido, del tipo temático categórico. Resultados: Se identificaron las siguientes categorías: Categoría I - Interferencia y convivencia con enfermedad mental y Categoría II - Carga en el cuidado de los enfermos mentales. Conclusión: Se considera que aún quedan vacíos por llenar para poder realizar una oferta calificada a estos actores (usuarios / cuidadores-familia) que, de hecho, puedan empoderar a estos sujetos y minimizar su sufrimiento.

Palabras claves: Sufrimiento; Salud mental; Precaución; Familia.

\section{Aline Pereira da Silva}

Enfermeira. Pós-graduada em obstetrícia e neonatologia. Universidade Federal de Campina Grande.

ORCID: 0000-0001-8185-9471

\section{Perla Figueredo Carreiro Soares}

Enfermeira. Mestre em Neurociência Cognitiva e Comportamento. Pós-graduada em enfermagem obstétrica. Chefe do Núcleo de Serviços Diagnósticos/SES/PB. ORCID: 0000-0002-0407-685X

\section{Erlânia Souza Costa}

Enfermeira. Pós-graduada em obstetrícia e neonatologia. Faculdade de Enfermagem Nova Esperança.

ORCID: 0000-0002-5018-0404

\section{Lúcia Gomes de Souza Silva}

Enfermeira. Faculdade Maurício de Nassau. Pós-graduada em Urgência/Emergência e Unidade de Terapia Intensiva.

ORCID: 0000-0001-6998-435X

\section{Rogéria Gomes da Silva}

Enfermeira. Pós-graduada em Gestão em Saúde, Gestão da Clínica nas regiões de Saúde e Docência do Ensino Superior. Chefe do Núcleo de Atenção Ambulato$\mathrm{rial} / \mathrm{SES} / \mathrm{PB}$.

ORCID: 0000-0001-7075-1369

\section{Luanna Silva Braga}

Enfermeira. Mestre em Enfermagem pelo Programa de Pós-Graduação em Enfermagem da Universidade Federal da Paraíba (PPGENF/UFPB).

ORCID: 0000-0002-0093-0406

Recebido em: 16/06/2021

Aprovado em: 15/07/2021
INTRODUÇÃO<smiles>C[C@@H]1C[C@H]2C[C@H]1C2</smiles>
prevalência de indivíduos acometidos por sofrimento psíquico na população em geral é bastante significativa, segundo dados do Ministério da Saúde (MS) 3\% da população em geral sofre de transtorno mental severo persistente; mais de $6 \%$ da população apresenta transtorno psiquiátrico grave decorrente do uso de álcool e drogas; $12 \%$ da população necessita de algum atendimento em saúde mental, seja ele contínuo ou eventual, sendo que somente $2,3 \%$ do orçamento anual do SUS é destinado para a saúde mental ${ }^{(1)}$.

A Reforma Psiquiátrica Brasileira tem por base legal a lei 10.216 criada pelo deputado Paulo Delgado, sancionada em 2001, que redireciona a assistência em saúde mental, privilegiando o ofereci- 
mento de tratamento em serviços de base comunitária sobre a proteção e os direitos das pessoas em sofrimento psíquico, mas não estabelece mecanismos claros para a diminuição dos manicômios. Embora a promulgação desse documento imponha novo impulso e novo ritmo para o processo de desistitucionalização( ${ }^{(2)}$.

Atualmente, a política nacional de saúde mental brasileira tem como base os princípios do Sistema Único de Saúde (SUS) e as diretrizes da reforma psiquiátrica, que preconiza o resgate a cidadania do sofrimento psíquico. Nesse sentido, torna-se indispensável à presença e o apoio da família para efetivação da assistência ao transtorno mental, tendo em vista que as primeiras manifestações de mudanças comportamentais são percebidas no ambiente familiar ${ }^{(2)}$.

Como base nesses novos paradigmas decorrentes da Reforma Psiquiátrica surgem os Centros de Atenção Psicossocial (CAPS). A criação desses centros possibilitou a organização de uma rede substitutiva ao Hospital Psiquiátrico no país. Os CAPS são serviços de saúde municipais, abertos, comunitários que oferecem atendimento diário. Estes serviços têm a função de substituir e não complementar o hospital psiquiátrico. De fato, o CAPS é o núcleo de uma nova clínica, produtora de autonomia, que convida o usuário à responsabilização e ao protagonismo em toda a trajetória do seu tratamento ${ }^{(1)}$.

Os projetos desses serviços, muitas vezes, ultrapassam a própria estrutura física, em busca da rede de suporte social, potencializadora de suas ações, preocupando-se com o sujeito e a singularidade, sua história, sua cultura e sua vida cotidiana. Outros demais dispositivos de atenção à saúde mental como o programa de volta para casa, residências terapêuticas têm importância estratégica para a Reforma Psiquiátrica Brasileira e a reinserção dos portadores de sofrimento psíquico na sociedade ${ }^{(1)}$.

Nesse momento de inclusão social, no contexto de acompanhamento e tratamento psicossocial, é de suma importância o oferecimento de suporte para a família em serviços extra-hospitalares, que sejam capazes de atender às necessi- dades da pessoa com sofrimento psíquico e sua família, de modo que esse processo ocorra o mais próximo possível da comunidade, valorizando a manutenção dos vínculos sociais e familiares ${ }^{(3)}$.

A família, em especial, o familiar/cuidador é submetido a constantes eventos estressores no curso dessa doença, que podem afetar, além das relações familiares, a saúde do próprio familiar/cuidador sempre trazendo algum grau de sobrecarga e provocando constante necessidade de adaptações ${ }^{(4)}$. Nesse sentindo, a responsabilização voltou-se mais para o seio da família, que ao se deparar com esse papel, tem a necessidade de encontrar novas estratégias de cuidado. Nesta perspectiva, cabe ressaltar a questão norteadora da pesquisa: Quais as estratégias de enfrentamento que o cuidador do doente mental desenvolve para melhor cuidar de seu familiar? Tem-se por objetivo: analisar a percepção do cuidado a pessoa com sofrimento mental sob a ótica dos familiares.

\section{MÉTODO}

O presente estudo trata-se de uma pesquisa exploratório-descritiva com abordagem qualitativa. O método exploratório foi selecionado por apresentar maior analogia com o estudo abordado, de acordo com $\mathrm{Gil}^{(5)}$, a pesquisa exploratória tem como intuito desenvolver, elucidar e transformar conceitos e ideias, tendo em vista a formulação de problemas mais precisos ou hipóteses. Neste contexto, o uso da pesquisa descritiva permitiu ao pesquisador expandir sua experiência acerca de um grupo já definido pelo pesquisador, preocupando-se com a atuação prática.

No que se refere à abordagem qualitativa, segundo Richardson ${ }^{(6)}$, é caracterizada por ser uma pesquisa detalhada sobre um devido tema e constituída pelas situações apresentadas pelos entrevistados. O estudo foi desenvolvido em um Centro de Atenção Psicossocial (CAPS I), em abril, ano de 2014.

No ano da pesquisa, a população no CAPS I, que fica localizado em um munícipio da Paraíba, na região nordeste, era composta por 78 usuários acompanhados pela equipe, sendo que destes 30 a 40 são ativos e cerca de 20 usuários frequentam diariamente o serviço. No ano de 2021, a rede de atenção à saúde mental aumentou, e os serviços foram ampliados, possuindo: 68 CAPS I (serviço para 15 mil habitantes); nove CAPS II (70 mil habitantes); 05 CAPS III 24 horas (150 mil); seis CAPS AD para 70 mil habitantes; 09 CAPS AD III 24h (150 mil); 12 CAPS Infantojuvenis (70 mil); 14 Residências Terapêuticas; cinco Consultórios na Rua; quatro Unidades de Acolhimento; 20 leitos de Saúde Mental em Hospital Geral e 65 beneficiários do Programa de Volta Pra Casa (PVC) (7). O serviço no qual foi realizada a pesquisa passou por mudanças, e no ano de 2021 possui: 95 usuários monitorados pela equipe, e deste grupo, 40 estão ativos, porém, diariamente 25 estão vindo ao serviço, devido ao momento pandêmico vivenciado no Brasil e no mundo.

Continuamente, a amostra do estudo foi composta por cerca 20 familiares/ cuidadores de sofrimento psíquico. Essa amostra foi construída por acessibilidade. Para ser incluso na pesquisa, os participantes apresentaram os seguintes requisitos: ser familiar ou cuidador do usuário do Centro de Atenção Psicossocial (CAPS), o usuário deve estar participando ativamente do CAPS, o familiar ou cuidador de indivíduos em sofrimento psíquico deve estar disposto a participar voluntariamente da pesquisa, bem como assinar o terno de consentimento livre e esclarecido (TCLE). Portanto, foram excluídos da pesquisa todos os que não se enquadraram nesses critérios.

Como técnica para coleta e análise de dados foi realizada uma abordagem com os participantes através do grupo focal, que teve como objetivo a interação dos familiares/cuidadores de sofrimento psíquico de forma a promover uma ampla problematização sobre as estratégias de enfrentamento para o cuidado em saúde mental: percepção da família. Segundo Backes et a $\left.\right|^{(7)}$, o grupo focal configura-se como uma entrevista em grupo, na qual a interação é parte integrante do método. No processo, os encontros grupais possibilitaram aos participantes ex- 
plorarem seus pontos de vista, a partir de reflexões sobre uma determinada temática.

O material empírico foi coletado através da realização de um grupo focal com familiares e/ou cuidadores do usuário do CAPS, essa técnica possibilitou uma ampla problematização do problema abordado, para efetivação desse grupo focal elaboramos questões norteadoras e perguntas subjetivas, a fim de atingir os objetivos propostos no que se refere aos familiares ou cuidador de sofrimento psíquico com a utilização de um gravador (MP3), onde tivemos a possibilidade de escutar e ao mesmo tempo gravar o que foi dito no grupo.

O material empírico foi discutido através da técnica de Análise de conteúdo proposta por Bardin(8). Dessa forma utilizamos à análise de conteúdo tipo categorial-temática. Esta pesquisa seguiu a resolução 466/12 do Conselho Nacional de Saúde, sob o número do CAAE: 22372113.0.0000.5182, em Março, ano de 2014.

\section{RESULTADOS}

A amostra foi composta por 20 familiares/cuidadores, cerca de quinze participantes com faixa etária de 40 a 60 anos e genitora. Cinco participantes com faixa etária de 25 a 40 anos, irmã e tia. Analisou-se o conteúdo das falas dos participantes da pesquisa e depois de repetidas leituras do material transcrito, efetuou-se uma apreensão global dos conteúdos e, posteriormente, foram identificados os temas emergidos nas entrevistas. Abaixo, as categorias temáticas designadas.

Categoria Temática I - Interferência e convivência com a doença mental

$\mathrm{Na}$ pesquisa observamos através dos relatos que a interferência e as mudanças estão presentes no contexto da família, porém de formas distintas. Para alguns participantes o diagnóstico cedo do transtorno mental no ambiente familiar gera uma adaptação com as limitações e exigências imposta, ou seja, houve interferência, porém como a família passa muito tempo no cuidado as mudanças e interferências aca-

bam fazendo parte da rotina familiar. Os relatos que seguem enfatizam essa questão:

\begin{abstract}
"Não interfere, até porque a gente se acostuma com a situação, ele apresentou esse problema quando era criança ainda". (Participante 2)

"Não interfere, porque a gente aprende desde cedo a conviver com o problema". (Participante 4)
\end{abstract}

Outros participantes relatam que essa interferência e percebida de forma drástica. As participantes seguintes falaram dessa interferência da seguinte forma:

"Interfere na rotina, porque quase todos os meus horários estão voltado para ele, seja aqui no CAPS ou no dia-dia". (Participante 9)

"Interfere sim principalmente eu que trabalho, tem que estar participando constantemente das reuniões aqui no CAPS, até porque preciso saber como ele tá". (Participante 12)

As reuniões as quais o participante 9 e 12 se referem, trata-se de reuniões que o CAPS promove quinzenalmente com grupos de familiares, dessa forma possibilita uma interação e compartilhamento das vivências entre os participantes, constituindo um espaço de troca de conhecimento e de experiências. Os profissionais têm a oportunidade de orientar e esclarecer o familiar quanto às situações da vida cotidiana.

Desta forma, o Centro de Atenção Psicossocial (CAPS) tem papel fundamental no apoio a família e usuário. No CAPS, configuram-se como atividades de atenção à família o acoIhimento, a visita domiciliar, o atendimento individual e em grupo aos familiares, buscando, assim, implementar essa parceria e a interação entre os atores desse processo. Alguns entrevistados relataram em seus discursos o CAPS como estratégia de enfrentamento para as atribulações diárias e na efetivação do tratamento oferecido a seus familiares:

"Hoje interfere menos, antigamente a gente sofria demais ele era agressivo, vivia saindo de casa ele era internado direto, depois que ele começou a vir para o CAPS ele passou a tomar os remédios e as meninas aqui do CAPS ajudam muito". (Participante 19)

"Depois que ele começou a vir para o CAPS mudou demais, ele começou a tomar os remédios, o pessoal daqui ajuda muito logo aqui tem psicólogo, tem médico". (Participante 15)

As interferências causadas no cotidiano familiar divergem em circunstâncias entre familiares, apesar de alguns participantes referirem que a convivência com o portador de sofrimento psíquico no contexto familiar não causa interferências no cotidiano da família, podemos perceber na categoria seguinte que todos os participantes referem à sobrecarga no cuidado, subentende-se que existe a interferências, porém não percebida pelos participantes.

Categoria II - Sobrecarga no cuidado ao doente mental

Apesar de alguns familiares cuidadores negarem interferências no contexto familiar, todos os participantes da pesquisa afirmaram a existência da sobrecarga e do estresse, oriundos do cuidado ao sofrimento psíquico. A sobrecarga é atribuída ao excesso de trabalho e o estresse decorrente da difícil convivência com o sofrimento psíquico, sobretudo, em situações de grande dependência do indivíduo que recebe cuidado. Os depoimentos a seguir evidenciam a sobrecarga e o estresse no cotidiano familiar:

"Existe estresse e a sobrecarga, mas a sobrecarga ainda é maior, eu mesmo tenho que dar banho, tem que dar os medicamentos na mão dele e esperar ele tomar, tem que colocar ele para dormir e ainda trabalho fora, por isso acabo ficando sobrecarregada". (Participante 10). "Os dois existe, porque fico sobrecarregada com a quantidade de 
tarefas do dia a dia e às vezes não tenho tempo para resolver outros assuntos". (Participante 13)

"O estresse e a sobrecarga faz parte do dia-dia, já que tudo dele é comigo, tenho que ficar em cima pra ele comer, tomar banho e tantas outras coisas ai a gente acaba de estressando e perdendo a paciência". (Participante 14)

A sobrecarga familiar é compreendida em objetiva e subjetiva, a primeira se refere às consequências negativas concretas e observáveis resultantes do papel de cuidador, tais como perdas financeiras, perturbações na rotina da vida familiar, excesso de tarefas que o familiar deve executar no cuidado diário com o paciente e supervisões aos comportamentos problemáticos do paciente, sendo estes aspectos considerados como estímulos estressores para os familiares.

\section{DISCUSSÃO}

Uma vez que, de acordo com Grandi e Waidman $^{(10)}$ a convivência com o sofrimento psíquico no ambiente familiar é difícil, pois envolve muitas questões, tais como, o preconceito não só com seu familiar, mas com toda a família, a exclusão do familiar acometido pelo sofrimento psíquico, o medo e a vergonha pelos sintomas da doença, além do fato de que, nem sempre os membros familiares conseguem lidar com toda a diversidade e complexidade que envolve o transtorno mental, como, por exemplo, as mudanças na rotina da casa que incluem alterações nos horários das refeições, na lavagem das roupas, nas compras, nas atividades de recreação e lazer, entre outras.

Os Centros de Atenção Psicossocial (CAPS) constituem uma estratégia de lidar não apenas com pessoas em adoecimento mental, incluindo em suas ações a participação e colaboração dos familiares, amigos e cuidadores dos usuários que os frequentam como atores integrantes do processo ao oferecerem um leque de atividades voltadas para os usuários e seus familiares, garantem o atendimento, o tratamento e o acompa- nhamento com vistas à inclusão social e ao resgate da cidadania ${ }^{(11)}$.

A família é a instituição fundamental para a reabilitação do usuário junto à comunidade, na qual este está inserido. O envolvimento da família no tratamento dos usuários contribui para diminuir das recaídas e o número de internações psiquiátricas dos pacientes com transtorno mental severo. É primordial trabalhar com as famílias dos usuários, a fim de se resgatar a importância da não exclusão da pessoa em sofrimento psíquico do convívio familiar. Nesse sentido, torna-se imprescindível a participação do familiar no contexto da atenção psicossocial oferecida pelo Centro de Atenção Psicossocial (CAPS), desde sua inclusão na elaboração do plano terapêutico, nos grupos de familiares, nas reuniões e assembleias até o próprio suporte terapêutico que se faz necessário(11)

Para Cardoso; Galera; Vieira ${ }^{(4)}$, as consequências adversas do adoecimento mental de um familiar para as famílias vêm sendo sistematicamente documentadas e apontam para o fato de que todas as áreas do funcionamento familiar são afetadas pela presença da doença mental. É importante notar que a sobrecarga relacionada ao cuidado em saúde mental é um fenômeno facilmente perceptível e que persiste mesmo quando o paciente responde positivamente a tratamentos inovadores e efetivos.

Os autores acima supracitados ainda enfatizaram que esta sobrecarga pode estar atribuída a acúmulo de tarefas, aumento de custos financeiros, limitação das atividades cotidianas, fragilização dos relacionamentos entre os familiares, à percepção pessoal do familiar sobre a experiência de conviver com o doente, seus sentimentos quanto à responsabilidade e preocupações que envolvem o cuidado à sua saúde, a falta de autonomia do indivíduo em sofrimento psíquico é vista como um aspecto negativo que gera tensão e preocupação, afetando o cuidador emocionalmente.

A família sofre intensamente com a situação da pessoa adoecida, vivenciando sentimentos de aflição, depressão, isolamento, tristeza crônica, culpa e angústia. A presença do sofrimento psíquico provoca ruptura da rotina existencial da família, na qual o principal cuidador passa a colocar suas próprias necessidades e vontades em segundo plano, tornando-se sobrecarregado por arcar com os ônus gerados pela doença. Em vista disso, o modelo atual vigente da Rede de Atenção Psicossocial tem como meta a reinclusão das pessoas com sofrimento psíquico, na comunidade e na família, através da recuperação da autoestima e reestruturação de vínculos, oferecendo apoio à família no intuito de amenizar o sofrimento tanto do usuário quanto do cuidador ${ }^{(11)}$.

A sobrecarga subjetiva se refere à percepção ou avaliação pessoal dos familiares sobre a situação, envolvendo sua reação emocional e seu sentimento de estar sofrendo uma sobrecarga, atribuída por eles ao papel de cuidador. Refere-se ao grau em que os familiares percebem os comportamentos ou a dependência dos pacientes como fonte de preocupação ou tensão psicológica ${ }^{(10)}$.

O novo modelo de assistência ao portador de sofrimento psíquico promoveu a desistitucionalização é a reintegração do individuo em sofrimento psíquico na família e na comunidade, no entanto existem divergências no que concerne o convívio do sofrimento psíquico no ambiente familiar. A doença mental representa impacto para a família ocasionando desorganizações nas formas habituais de lidar com situações do cotidiano, na medida em que a família enfrenta as alterações de comportamento do familiar e passa a assumir a função do cuidador, a responder às demandas desta função ${ }^{(10)}$.

Todas as áreas de funcionamento familiar são afetadas pela presença do sofrimento psíquica à ponta de criar estratégias de enfrentamento para amenizar o estresse e a sobrecarga atribuída ao cuidado ofertado ao seu familiar em sofrimento psíquico. Diante desse cenário os serviços extra-hospitalares devem munir as famílias de informação pertinente à patologia, sintomatologia da doença, conhecer sua história, cultura, crenças, valores, hábitos e costumes para que dessa forma possa 
amenizar a sobrecarga dos familiares e resgatar a dignidade e autonomia do indivíduo em sofrimento psíquico ${ }^{(11)}$

A rede de atenção à saúde mental vem funcionando de novas formas, com estratégias tidas como mais efetivas. Com o passar dos anos, o progresso de desinstitucionalização se tornaram significativos, houve redução de leitos psiquiátricos de 53.962 em 2001 para 25.988 em $2014^{(12-}$ 13). Esse processo foi iniciado desde 2001 com a implantação da lei que defendia os direitos das pessoas em sofrimento mental, mas demorou 13 anos para começar a se ter resultados aparentes ${ }^{(12-13)}$.

Em 2015, após a realização da pesquisa, um estudo demonstrou que o Brasil possui representantes de vários setores da saúde mental, e que as produções científicas na temática cresceram, e que trouxeram maior qualidade para a assistência. Entretanto, ficou evidenciado que ainda existem insuficiências no sistema de saúde, e que as necessidades dos pacientes e seus familiares em alguns momentos não são supridas, como é o caso da atenção ás urgências. Em 2016, os governantes repensaram nas estratégias, houve a ampliação de alguns serviços $^{(12-13)}$, como por exemplo, o CAPS, no qual percebemos que passou a ter este serviço em outros munícipios na paraíba, atendendo mais usuários. Percebe-se que modelo hospitalocêntrico precisa ser supe- rado, e que a todo momento surgem novos serviços para substituir este processo, sendo a Atenção Primária à Saúde um dos setores que também pode prestar assistência em saúde mental com qualidade ${ }^{(14)}$.

\section{CONCLUSÃO}

Ao analisamos as percepções dos familiares acerca do cuidado ao sofrimento psíquico e as prováveis repercussões deste cuidado em suas vidas, percebemos a importância da família no tratamento, acompanhamento e desenvolvimento do indivíduo em sofrimento psíquico no contexto familiar e na reinserção social. A família é principal elo de comunicação no contexto social.

O processo de desistitucionalização incluiu a família como parte integrante do tratamento ao sofrimento psíquico, no entanto demandou maior responsabilidade dos familiares acerca do cuidado oferecido em sofrimento psíquico, acarretando dessa forma mudanças bruscas no cotidiano da família, gerando sobrecarga e estresse no contexto familiar.

O cuidado em saúde mental perpassou por avanços, conforme visto na discussão desta pesquisa, e acarretou no cotidiano das famílias um impacto, no que diz respeito às adaptações decorrente da convivência do sofrimento mental com os demais integrantes da família. Esta por sua vez, passa a garantir as necessidades básicas do familiar doente, essas atribuições geram no cuidador sentimentos de angustia e aflição.

No que concerne às estratégias elaboradas por estes familiares que ajude a atenuar a sobrecarga e, consequentemente, promover uma melhor assistência no cuidado ao portador de sofrimento psíquico, foi identificado nas análises que os cuidadores/familiares não planejam ou não conseguem identificar as estratégias utilizadas para efetivar o cuidado ao portador de sofrimentos. No entanto, na falta de planejamento, organização das ideias e no enfrentamento das dificuldades diárias, a maioria dos participantes verbalizou que a fé e o CAPS como sendo a principal força para superar as atribulações diárias.

Por todos estes aspectos, vale ressaltar a importância do Centro de Atenção Psicossocial (CAPS) no que diz respeito ao apoio e cuidado aos familiares e usuários destes serviços, sendo imperativo que estes serviços devam acolher o sofrimento das famílias e minimizar sua sobrecarga emocional por meio da oferta de espaços acolhedores e facilitadores de ações e de troca de experiências entre os próprios cuidadores, para compartilhar dúvidas, angústias e alegrias em um movimento de proximidade com o tratamento e em direção à autonomia dos pacientes e à diminuição do sofrimento das famílias.

\section{Referências}

1. Brasil. Ministério da Saúde. Saúde mental. 2013.

2. Brasil. Ministério da Saúde. Secretaria de Atenção à Saúde. DAPE: Coordenação Geral de Saúde Mental. Reforma psiquiátrica e políticas de saúde mental no Brasil. Documento apresentado à Conferência Regional de Reforma dos Serviços de Saúde Mental: 15 anos depois de Caracas. OPAS. Brasília, novembro de 2005.

3. Gonçalves, JRL; Luiz, MAV. Atendimento ao familiar cuidador em convívio com o portador de transtorno mental. Revista de Enfermagem UERJ. Rio de Janeiro, v. 18, n. 2, p. 272-277, abr./jun. 2010.

4. Cardoso, L; Galera, SAF; Vieira, MV. 0 cuidado e a sobrecarga do cuidado à saúde de pacientes egressos de internação psiquiátrica. Acta Paulista de Enfermagem. São Paulo, v. 25, n. 4, p. 23-517, 2012.

5. Gil, AC. Como elaborar projetos de Pesquisas. 4 ed. São Paulo: Atlas, 2008.

6. Richardson, RJ. et al. Pesquisa Social: métodos e técnicas. 3. ed. revista e ampliada. São Paulo: Atlas, 2008.

7. Paraíba. Monitoramento de saúde Mental. 2021.

8. Backes, DS. et al. Grupo focal como técnica de coleta e análise de dados em pesquisas qualitativas. 0 Mundo da Saúde, v. 35, n. 4, p. 438-442, 2011.
Bardin, L. Análide de conteúdo. 2006.

9. Grandi, AL.; Waidman, MAP. Convivência e rotina da família atendida em CAPS. Ciência, Cuidado e Saúde, v. 10, n. 4, p. 763-772, 2012.

10. Carvalho, MAP. de et al. Contribuições da terapia comunitária integrativa para usuários dos Centros de Atenção Psicossocial (CAPS): do isolamento à sociabilidade libertadora Contributions by integrative community therapy. Cadernos de Saúde Pública, v. 29, n. 10, p. 2028-2038, 2013.

11. Gomes, MS.; Mello, R. Sobrecarga gerada pelo convívio com o portador de esquizofrenia: enfermagem construindo o cuidado à família. SMAD - Revista eletrônica saúde mental, álcool e drogas, v. 8, n. 1, p. 2-8, 2012.

12. Almeida, JMC de. Espaço temático: saúde mental no brasil: avanços e retrocessos. Cad. Saúde Pública 35 (11) 31 Out 2019.

13. Alves Maftum M, Gonçalves da Silva Ângela, de Oliveira Borba L, Brusamarello T, Czarnobay J. Mudanças ocorridas na prática profissional na área da saúde mental frente à reforma psiquiátrica brasileira na visão da equipe de enfermagem. R. pesq. cuid. fundam. Online.;9(2):309-14.

14. Silva Filho, JAda; Silva, CRL da; Marques, APBF; Nóbrega, RJN; Pinto, AGA. Nursing (Säo Paulo); 23(262): 3638-3642, mar.2020. 